\section{REFERENCES}

1. Chowaniec M, Starba A, Wiland P. Erythema nodosum review of the literature. Reumatologia 2016;54:79-82.

2. Matsuoka LY. Neoplastic erythema nodosum. J Am Acad Dermatol 1995;32(2 Pt 2):361-363.

3. Virshup AM, Sliwinski AJ. Polyarthritis and subcutaneous nodules associated with carcinoma of the pancreas. Arthritis
Rheum 1973;16:388-392.

4. Durden FM, Variyam E, Chren MM. Fat necrosis with features of erythema nodosum in a patient with metastatic pancreatic carcinoma. Int J Dermatol 1996;35:39-41.

5. Arbeláez-Cortés A, Vanegas-García AL, Restrepo-Escobar M, Correa-Londoño LA, González-Naranjo LA. Polyarthritis and pancreatic panniculitis associated with pancreatic carcinoma: review of the literature. J Clin Rheumatol 2014;20:433-436.

\title{
Repigmentation of Eyebrow Leukotrichia in Segmental Vitiligo Treated with Suction Blister Epidermal Grafting Following Hair Plucking
}

\author{
Se Jin Oh, Cho Rok Kim ${ }^{1}$, Ji-Hye Park, Dong-Youn Lee, Dokyoung Yoon \\ Department of Dermatology, Samsung Medical Center, Sungkyunkwan University School of Medicine, ${ }^{1}$ Kye Dermatology Clinic, Seoul, Korea
}

\begin{abstract}
Dear Editor:
Vitiligo is generally classified into two clinical categories, nonsegmental vitiligo and segmental vitiligo (SV) ${ }^{1}$. SV on hairy areas such as the scalp and eyebrows is frequently associated with leukotrichia ${ }^{2}$. SV with overlying leukotrichia is refractory to conventional medical treatments because leukotrichia itself suggests a deficient melanocyte reservoir within the hair follicles ${ }^{1}$. Thus surgical management including epidermal grafting should be considered and preferred as an early intervention for the SV with leukotrichia, however only few literature showed improvement following surgical treatment. Herein, we present two cases of SV on the eyebrow successfully treated with suction blister epidermal grafting (SBEG) after hair plucking. An 11-year-old boy showed SV and leukotrichia on his left eyelid and forehead which had developed 8 months ago
\end{abstract}

(Fig. 1A). A 16-year-old girl had SV with leukotrichia on her right eyebrow and eyelid (Fig. 2A). Both cases did not respond to 6 months of conventional combination treatment including 308-nm excimer laser, topical steroid, and a calcineurin inhibitor. SBEG was performed under the consent of both the patients. Hairs of the eyebrow were pulled with forceps as a whole, including the hair follicle. Thigh skin was used as a donor for epidermal grafting. Vacuum suction was performed to donor sites at a pressure of 200 to $250 \mathrm{mmHg}$, and blisters formed after 2 hours. Epidermis on the recipient site was removed with defocusing, superpulsed mode $\mathrm{CO}_{2}$ laser. Subsequently, the roofs of the bullae were carefully excised and grafted. The recipient areas were almost completely pigmented and leukotrichia was significantly improved after $1 \sim 2$ years of clinical follow-up (Fig. 1B, 2B).

\footnotetext{
Received March 13, 2019, Revised May 9, 2019, Accepted for publication June 3, 2019
}

Corresponding author: Dong-Youn Lee, Department of Dermatology, Samsung Medical Center, Sungkyunkwan University School of Medicine, 81 Irwon-ro, Gangnam-gu, Seoul 06351, Korea. Tel: 82-2-3410-6578, Fax: 82-2-3410-3869, E-mail: dylee@skku.edu ORCID: https://orcid.org/0000-0003-0765-9812

This is an Open Access article distributed under the terms of the Creative Commons Attribution Non-Commercial License (http://creativecommons.org/licenses/by-nc/4.0) which permits unrestricted non-commercial use, distribution, and reproduction in any medium, provided the original work is properly cited.

Copyright (c) The Korean Dermatological Association and The Korean Society for Investigative Dermatology 

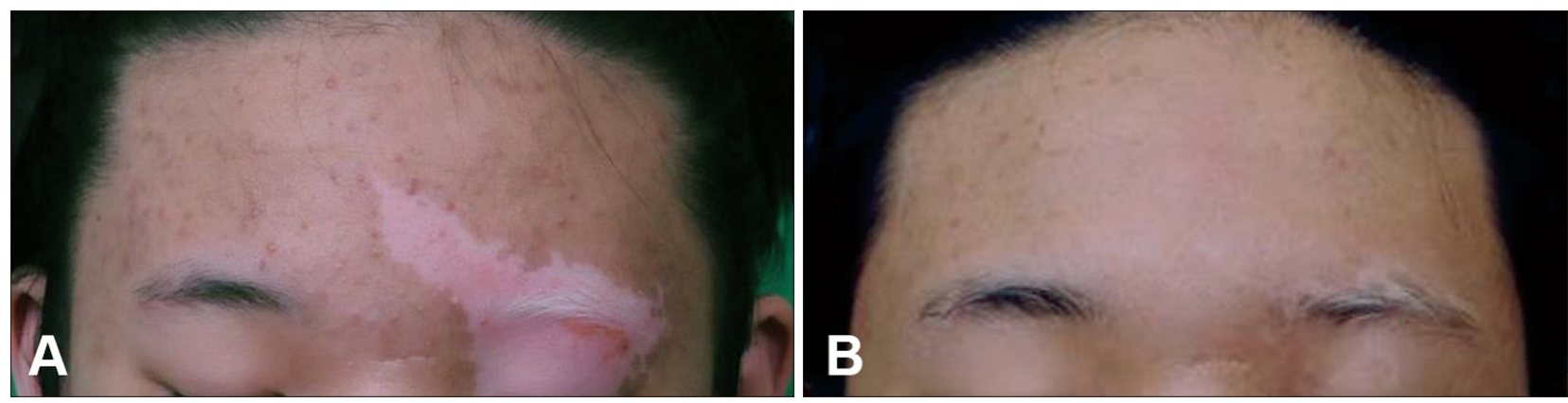

Fig. 1. (A) Well-defined depigmented patches on left side of forehead and eyelid with leukotrichia of the eyebrow. (B) After 2 years of treatment showing successful repigmentation of white hairs. We received the patient's consent form about publishing all photographic materials.
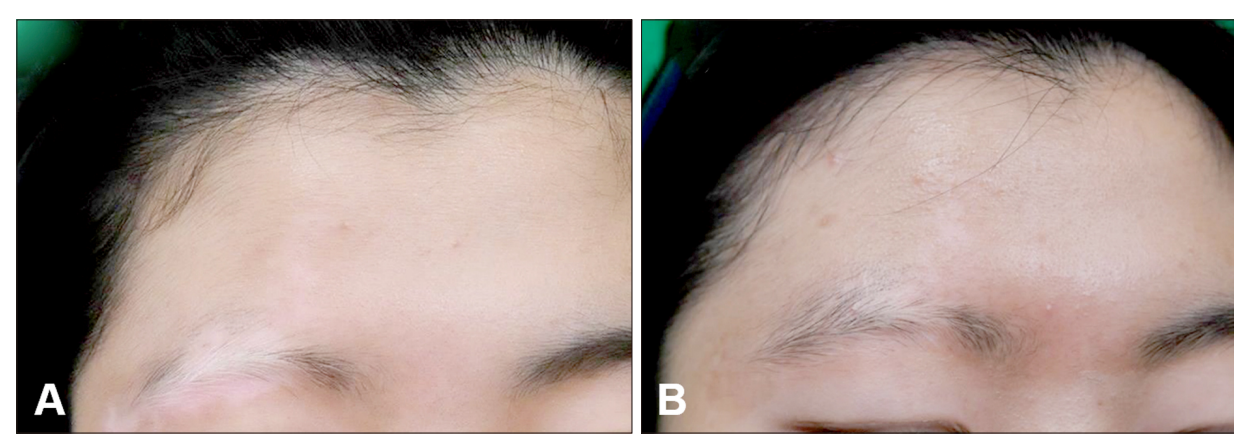

Fig. 2. (A) Whitish patches on the right side of the eyelid with leukotrichia of the eyebrow. (B) After 1 year of treatment showing successful repigmentation of white hairs. We received the patient's consent form about publishing all photographic materials.
Surgical approaches are required for SV with leukotrichia because the obliteration of melanocyte reservoir within the hair follicles is persistent ${ }^{3}$.

In this case, $\mathrm{CO}_{2}$ laser resurfacing technique was applied to the recipient sites. Rapid, uniform and reproducible deepithelization can be achieved with a single laser pass yielding minimal thermal damage ${ }^{4}$. Also, suction blister method was used to harvest the donor, which is known to be easy, safe, inexpensive, and effective ${ }^{5}$.

According to the previous study, melanocytes migrate from epidermis to hair follicle retrogradely and this process leads to improvement of leukotrichia ${ }^{1}$. If the hair on the recipient site remains and grows, this hair can push out the grafted epidermal sheet and thus disturb the movement of the melanocytes. In our case, we formed empty space through hair plucking and lead to successful migration of melanocytes and also repigmentation of leukotrichia. Among the three common hair removal methods, razor shaving, chemical epilation, and hair plucking can be the best option, which is simple and moreover rarely induces procedure-related complications. Razor shaving is easy to perform but it is difficult to create an empty space because the hair can be incompletely removed. Chemical epilation is painless, but thioglycolic acid, a major component, is a common cause of irritant contact dermatitis and moreover can leave a post-inflammatory hyperpigmentation as well.
In conclusion, we suggest that SBEG following hair plucking can be an effective treatment option for patients with leukotrichia in SV.

\section{CONFLICTS OF INTEREST}

The authors have nothing to disclose.

\section{ORCID}

Se Jin Oh, https://orcid.org/0000-0001-7525-4740

Cho Rok Kim, https://orcid.org/0000-0003-4168-4245

Ji-Hye Park, https://orcid.org/0000-0002-6699-5202

Dong-Youn Lee, https://orcid.org/0000-0003-0765-9812

Dokyoung Yoon, https://orcid.org/0000-0002-1769-4921

\section{REFERENCES}

1. Holla AP, Sahni K, Kumar R, Kanwar A, Mehta S, Parsad D. Repigmentation of leukotrichia due to retrograde migration of melanocytes after noncultured epidermal suspension transplantation. Dermatol Surg 2014;40:169-175.

2. Kim CY, Yoon TJ, Kim TH. Epidermal grafting after chemical epilation in the treatment of vitiligo. Dermatol Surg 2001; 27:855-856.

3. Lee DY, Kim CR, Park JH, Lee JH. The incidence of leuko- 
trichia in segmental vitiligo: implication of poor response to medical treatment. Int J Dermatol 2011;50:925-927.

4. Al-Hadidi N, Griffith JL, Al-Jamal MS, Hamzavi I. Role of recipient-site preparation techniques and post-operative wound dressing in the surgical management of vitiligo. J
Cutan Aesthet Surg 2015;8:79-87.

5. Nanda S, Relhan V, Grover C, Reddy BS. Suction blister epidermal grafting for management of eyelid vitiligo: special considerations. Dermatol Surg 2006;32:387-391; discussion 391-392. 